\title{
Solvability of impulsive partial neutral second-order functional integro-differential equations with infinite delay
}

Shengli Xie*

"Correspondence:

slxie@ahjzu.edu.cn; xieshengli200@sina.com

Department of Mathematics and Physics, Anhui University of Architecture, Zipeng Road, Hefei, Anhui 230601, P.R. China

\begin{abstract}
Using the Kuratowski measure of noncompactness and progressive estimation method, we obtain the existence results of mild solutions for impulsive partial neutral second-order functional integro-differential equations with infinite delay in Banach spaces. The compactness condition of the impulsive term, some restrictive conditions on a priori estimation and noncompactness measure estimation have been deleted. Our conditions are simple and our results essentially improve and extend some known results. As applications, some examples are provided to illustrate the obtained results.
\end{abstract}

MSC: 34K30; 34K40; 35R10; 47D09

Keywords: impulsive partial neutral functional integro-differential equations; mild solutions; fixed point; Banach spaces

\section{Introduction}

Consider the following impulsive partial neutral second-order functional integro-differential systems with infinite delay in a Banach space $X$ :

$$
\begin{aligned}
& \left\{\begin{array}{l}
\frac{d}{d t}\left[x^{\prime}(t)+g_{1}\left(t, x_{t}, \int_{0}^{t} k_{1}\left(t, s, x_{s}\right) d s\right)\right]=A x(t)+g_{2}\left(t, x_{t}, \int_{0}^{t} k_{2}\left(t, s, x_{s}\right) d s\right), \\
\quad t \in[0, b], \\
\Delta x\left(t_{i}\right)=I_{i}^{1}\left(x_{t_{i}}\right), \quad \Delta x^{\prime}\left(t_{i}\right)=I_{i}^{2}\left(x_{t_{i}}\right), \quad i=1,2, \ldots, n, \\
x_{0}=\varphi \in \beta, \quad x^{\prime}(0)=z \in X,
\end{array}\right. \\
& \left\{\begin{array}{l}
\frac{d}{d t}\left[x^{\prime}(t)+g\left(t, x_{t}, x_{t}^{\prime}\right)\right]=A x(t)+\int_{0}^{t} f\left(s, x_{s}, x_{s}^{\prime}\right) d s, \quad t \in[0, b], \\
\Delta x\left(t_{i}\right)=I_{i}^{1}\left(x_{t_{i}}, x_{t_{i}}^{\prime}\right), \quad \Delta x^{\prime}\left(t_{i}\right)=I_{i}^{2}\left(x_{t_{i}}, x_{t_{i}}^{\prime}\right), \quad i=1,2, \ldots, n, \\
x_{0}=\varphi \in \beta, \quad x_{0}^{\prime}=\psi \in \beta,
\end{array}\right.
\end{aligned}
$$

where $A$ is the infinitesimal generator of a strongly continuous cosine function of bounded linear operators, $(C(t))_{t \in \mathbb{R}}$, on $X$. In both cases, the history $x_{t}, x_{t}^{\prime}:(-\infty, 0] \rightarrow X, x_{t}(\theta)=$ $x(t+\theta)$ and $x_{t}^{\prime}(\theta)=x^{\prime}(t+\theta)$ belongs to some abstract phase space $\beta$ defined axiomatically; $g, f, g_{j}, k_{j}, I_{i}^{j}(j=1,2)$ are appropriate functions; $0=t_{0}<t_{1}<\cdots<t_{n}<t_{n+1}=b$ are fixed numbers and the symbol $\triangle x\left(t_{i}\right)$ represents the jump of the function $x$ at $t_{i}$, which is defined by $\triangle x\left(t_{i}\right)=x\left(t_{i}^{+}\right)-x\left(t_{i}^{-}\right), i=1,2, \ldots, n$. 
The study of impulsive functional differential equations is linked to their utility in simulating processes and phenomena subject to short-time perturbations during their evolution. The perturbations are performed discretely and their duration is negligible in comparison with the total duration of the processes and phenomena. Now impulsive partial neutral functional differential equations have become an important object of investigation in recent years stimulated by their numerous applications to problems arising in mechanics, electrical engineering, medicine, biology, ecology, etc. With regard to this matter, we refer the reader to [1-12] and references therein. However, in order to obtain the existence of solutions in these study papers, the compactness condition on the associated family of operators and the impulsive term, some similar restrictive conditions on a priori estimation,

$$
\begin{aligned}
& \mu=K_{b} \sum_{i=1}^{n}\left(N c_{i}^{1}+\bar{N} c_{i}^{2}\right)<1, \quad \frac{K_{b}}{1-\mu} \int_{0}^{t}\left(N m_{g}(s)+\bar{N} m_{f}(s)\right) d s<\int_{c}^{\infty} \frac{d s}{W(s)}, \\
& K_{b}\left[N L_{g} b+\sum_{i=1}^{n}\left(N L_{i}^{1}+\bar{N} L_{i}^{2}\right)+\bar{N} \liminf _{\xi \rightarrow+} \frac{W_{f}(\xi)}{\xi} \int_{0}^{t} m_{f}(s)\right] d s<1,
\end{aligned}
$$

are used. In [13-16], authors used a strict set contraction mapping fixed point theorem without the compactness assumption on the associated family of operators to obtain the existence results of system (1) when $g_{i}\left(t, x_{t}\right)$ is not an integral operator and the following system:

$$
\left\{\begin{array}{l}
\frac{d}{d t}\left[x^{\prime}(t)+g\left(t, x_{t}, x^{\prime}(t)\right)\right]=A x(t)+f\left(t, x_{t}, x^{\prime}(t)\right), \quad t \in[0, b], t \neq t_{i}, \\
\Delta x\left(t_{i}\right)=I_{i}^{1}\left(x_{t_{i}}, x^{\prime}\left(t_{i}\right)\right), \quad \Delta x^{\prime}\left(t_{i}\right)=I_{i}^{2}\left(x_{t_{i}}, x^{\prime}\left(t_{i}\right)\right), \quad i=1,2, \ldots, n, \\
x_{0}=\varphi \in \beta, \quad x^{\prime}(0)=z,
\end{array}\right.
$$

improved and generalized some results in $[1,7]$. However, the compactness condition of the impulsive terms $I_{i}^{j}(\cdot)$, some similar restrictive conditions on a priori estimation (3), (4) and the restrictive condition on measure of noncompactness estimation

$$
K_{b}\left[N L_{g} b+\sum_{i=1}^{n}\left(N L_{i}^{1}+\tilde{N} L_{i}^{2}\right)\right]+\int_{0}^{b} \eta(s) d s<1
$$

are used in [13-16]. So far we have not seen the existence results of system (2).

In this paper, using the Kuratowski measure of noncompactness and progressive estimation method, we obtain the existence results of mild solutions of impulsive partial neutral second-order functional integro-differential systems (1) and (2). The compactness condition of impulsive terms $I_{i}^{j}(\cdot)$, some restrictive conditions on a priori estimation and measure of noncompactness estimation (3), (4) and (6) have been deleted. Our conditions are simple and our results essentially improve and extend some corresponding results in papers $[1,2,13,14]$. As applications, some examples are provided to illustrate the obtained results.

\section{Preliminaries}

In this paper, $X$ is a Banach space with the norm $\|\cdot\|$ and $A$ is the infinitesimal generator of a strongly continuous cosine function of bounded linear operators, $(C(t))_{t \in \mathbb{R}}$, on $X$ and 
$S(t)$ is the sine function associated with $(C(t))_{t \in \mathbb{R}}$, which is defined by $S(t) x=\int_{0}^{t} C(s) x d s$, $x \in X, t \in \mathbb{R}$. We designate by $N, \bar{N}$ certain constants such that $\|C(t)\| \leq N$ and $\|S(t)\| \leq$ $\bar{N}$ for every $t \in J=[0, b]$. We refer the reader to [17] for the necessary concepts about cosine functions. Next, we only mention a few results and notations needed to establish our results. As usual we denote by $[D(A)]$ the domain of $A$ endowed with the graph norm $\|x\|_{A}=\|x\|+\|A x\|, x \in D(A)$. Moreover, the notation $E$ stands for the space formed by the vector $x \in X$, for which the function $C(\cdot) x$ is of class $C^{1}$. It was proved by Kisyński [18] that the space $E$ endowed with the norm

$$
\|x\|_{E}=\|x\|+\sup _{0 \leq t \leq b}\|A S(t) x\|, x \in E,
$$

is a Banach space. The operator-valued function $G(t)=\left[\begin{array}{cc}C(t) & S(t) \\ A S(t) & C(t)\end{array}\right]$ is a strongly continuous group of linear operators on the space $E \times X$ generated by the operator $\mathcal{A}=\left[\begin{array}{ll}0 & I \\ A & 0\end{array}\right]$ defined on $D(A) \times E$. It follows from this that $A S(t): E \rightarrow X$ is a bounded linear operator and that $A S(t) x \rightarrow 0(t \rightarrow 0)$ for each $x \in E$. Furthermore, if $x:[0, \infty) \rightarrow X$ is a locally integrable function, then $z(t)=\int_{0}^{t} S(t-s) x(s) d s$ defines an $E$-valued continuous function. This is a consequence of the fact that

$$
\int_{0}^{t} G(t-s)\left[\begin{array}{c}
0 \\
x(s)
\end{array}\right] d s=\left[\begin{array}{c}
\int_{0}^{t} S(t-s) x(s) d s \\
\int_{0}^{t} C(t-s) x(s) d s
\end{array}\right]
$$

defines an $(E \times X)$-valued continuous function. Next, we denote $N_{1}=\sup _{t \in J}\|A S(t)\|_{L(E, X)}$, in which $L(E, X)$ stands for the Banach space of bounded linear operators from $E$ into $X$, and we abbreviate this notation to $L(X)$ when $E=X$.

To describe appropriately our system (1), we say that the function $u:[\sigma, \tau] \rightarrow X$ is a normalized piecewise continuous function on $[\sigma, \tau]$ if $u$ is piecewise continuous and left continuous on $(\sigma, \tau]$. We denote by $P C([\sigma, \tau], X)$ the space formed by the normalized piecewise continuous functions from $[\sigma, \tau]$ into $X$. In particular, we introduce the space $P C$ formed by all functions $u:[0, b] \rightarrow X$ such that $u$ is continuous at $t \neq t_{i}$, $u\left(t_{i}^{-}\right)=u\left(t_{i}\right)$ and $u\left(t_{i}^{+}\right)$exists for all $i=1,2, \ldots, n$. It is clear that $P C$ endowed with the norm $\|x\|_{p c}=\sup _{t \in J}\|x(t)\|$ is a Banach space.

For $x \in P C$, let

$$
\tilde{x}_{i}(t)= \begin{cases}x(t), & t \in\left(t_{i}, t_{i+1}\right] \\ x\left(t_{i}^{+}\right), & t=t_{i}, i=0,1, \ldots, n .\end{cases}
$$

Then $\tilde{x} \in C\left(\left[t_{i}, t_{i+1}\right], X\right)$. Moreover, for $V \subseteq P C$ and $i=0,1, \ldots, n$, we use the notation $\widetilde{V}_{i}$ for $\widetilde{V}_{i}=\left\{\tilde{x}_{i}: x \in V\right\}$. From Lemma 1.1 in [1], we know that a set $V \subseteq P C$ is relatively compact if and only if each set $\widetilde{V}_{i}=\left\{\widetilde{x}_{i}: x \in V\right\}$ is relatively compact in $C\left(\left[t_{i}, t_{i+1}\right], X\right)(i=0,1, \ldots, n)$.

For system (2), we give the precise meaning of the derivative in (2). We say that $x \in$ $P C$ is piecewise smooth if $x$ is continuously differentiable at $t \neq t_{i}, i=1,2, \ldots, n$, and for $t=t_{i}, i=1,2, \ldots, n$, there are the right derivative $x^{\prime}\left(t_{i}^{+}\right)=\lim _{s \rightarrow 0+} \frac{x\left(t_{i}+s\right)-x\left(t_{i}\right)}{s}$ and the left derivative $x^{\prime}\left(t_{i}^{-}\right)=\lim _{s \rightarrow 0-} \frac{x\left(t_{i}+s\right)-x\left(t_{i}\right)}{s}$. Furthermore, we denote the space by $P C^{1}=\{x \in P C$ : $x^{\prime}(t)$ is continuous at $t \neq t_{i}, x^{\prime}\left(t_{i}^{-}\right)$and $x^{\prime}\left(t_{i}^{+}\right)$exist, $\left.i=1,2, \ldots, n\right\}$. Then $P C^{1}$ endowed with the norm $\|u\|_{1}=\|u\|_{p c}+\left\|u^{\prime}\right\|_{p c}$ is a Banach space. 
In this work we employ an axiomatic definition of the phase space $\beta$ introduced by Hale and Kato [19] which appropriated to treat retarded impulsive differential equations. For other abstract phase spaces, we can refer to [20,21].

Definition $2.1[19]$ The phase space $\beta$ is a linear space of functions mapping $(-\infty, 0]$ into $X$ endowed with a seminorm $\|\cdot\|_{\beta}$. We assume that $\beta$ satisfies the following axioms.

(A) If $x:(-\infty, \sigma+b] \rightarrow X(b>0)$ is such that $x_{\sigma} \in B$ and $\left.x\right|_{[\sigma, \sigma+b]} \in P C([\sigma, \sigma+b], X)$, then for every $t \in[\sigma, \sigma+b)$ the following conditions hold:

(i) $x_{t}$ is in $\beta$,

(ii) $\|x(t)\| \leq H\left\|x_{t}\right\|_{\beta}$,

(iii) $\left\|x_{t}\right\|_{B} \leq K(t-\sigma) \sup \{\|x(s)\|: \sigma \leq s \leq t\}+M(t-\sigma)\left\|x_{\sigma}\right\|_{\beta}$, where $H>0$ is a constant; $K, M:[0, \infty) \rightarrow[1, \infty), K$ is continuous, $M$ is locally bounded and $H, K, M$ are independent of $x(\cdot)$.

(B) The space $B$ is complete.

In this paper we denote by $\alpha(\cdot)$ the Kuratowski measure of noncompactness of $X$, by $\alpha_{c}(\cdot)$ the Kuratowski measure of noncompactness of $C([0, b], X)$ and by $\alpha_{p c}(\cdot)$ the Kuratowski measure of noncompactness of $P C$.

The following lemma is easy to get.

Lemma 2.2 If the cosine function family $C(t), t \in \mathbb{R}$, is equicontinuous and $\eta \in L\left([0, b], \mathbb{R}^{+}\right)$, then the set

$$
\left\{\int_{0}^{t} S(t-s) u(s) d s,\|u(s)\| \leq \eta(s) \text { for a.e. } s \in[0, b]\right\}
$$

is equicontinuous for $t \in[0, b]$.

Lemma $2.3[16,22]$

(1) If $W \subset P C([0, b], X)$ is bounded, then $\alpha(W(t)) \leq \alpha_{p c}(W)$ for any $t \in[0, b]$, where $W(t)=\{u(t): u \in W\} \subseteq X$.

(2) If $W$ is piecewise equicontinuous on $[0, b]$, then $\alpha(W(t))$ is piecewise continuous for $t \in[0, b]$ and $\alpha_{p c}(W)=\sup \{\alpha(W(t)), t \in[0, b]\}$.

(3) If $W \subset P C([0, b], X)$ is bounded and piecewise equicontinuous, then $\alpha(W(t))$ is piecewise continuous for $t \in[0, b]$ and

$$
\alpha\left(\int_{0}^{t} W(s) d s\right) \leq \int_{0}^{t} \alpha(W(s)) d s, \quad \forall t \in[0, b]
$$

where $\int_{0}^{t} W(s) d s=\left\{\int_{0}^{t} x(s) d s: x \in W\right\}$.

(4) If $W \subset P C^{1}([0, b], X)$ is bounded and the elements of $W^{\prime}$ are equicontinuous on each $J_{i}=\left(t_{i}, t_{i+1}\right](i=0,1, \ldots, n)$, then

$$
\alpha_{p c^{1}}(W)=\max \left\{\sup _{t \in[0, b]} \alpha(W(t)), \sup _{t \in[0, b]} \alpha\left(W^{\prime}(t)\right)\right\}
$$

where $\alpha_{p c^{1}}(\cdot)$ denotes the Kuratowski measure of noncompactness in the space $P C^{1}([0, b], X)$. 
Lemma 2.4 [23] Let $h:[0, b] \rightarrow E$ be an integrable function such that $h \in P C$. Then the function $v(t)=\int_{0}^{t} C(t-s) h(s) d s$ belongs to $P C^{1}$, the function $s \rightarrow A S(t-s) h(s)$ is integrable on $[0, t]$ for $t \in[0, b]$ and

$$
v^{\prime}(t)=h(t)+A \int_{0}^{t} S(t-s) h(s) d s=h(t)+\int_{0}^{t} A S(t-s) h(s) d s, \quad t \in[0, b] .
$$

Lemma 2.5 [24] Let $V=\left\{x_{n}\right\} \subset L^{1}([a, b], X)$. If there is $\sigma \in L^{1}\left([a, b], \mathbb{R}^{+}\right)\left(\mathbb{R}^{+}=[0,+\infty)\right)$ such that $\left\|x_{n}(t)\right\| \leq \sigma(t)$ for $x \in V$ and a.e. $t \in[a, b]$, then $\alpha(V(t)) \in L^{1}\left([a, b], \mathbb{R}^{+}\right)$and

$$
\alpha\left(\left\{\int_{a}^{t} x_{n}(s) d s: n \in \mathbb{N}\right\}\right) \leq 2 \int_{a}^{t} \alpha(V(s)) d s, \quad t \in[a, b] .
$$

Lemma 2.6 [25] (Mónch) Let $X$ be a Banach space, $\Omega$ be a bounded open subset in $X$ and $0 \in \Omega$. Assume that the operator $F: \bar{\Omega} \rightarrow X$ is continuous and satisfies the following conditions:

(1) $x \neq \lambda F x, \forall \lambda \in(0,1), x \in \partial \Omega$,

(2) $D$ is relatively compact if $D \subset \overline{c o}(\{0\} \cup F(D))$ for any countable set $D \subset \bar{\Omega}$.

Then $F$ has a fixed point in $\bar{\Omega}$.

\section{Main results}

Firstly, we discuss the existence of mild solutions for the impulsive second-order system (1).

Definition 3.1 A function $x:(-\infty, b] \rightarrow X$ is said to be a mild solution of system (1) if $x_{0}=\varphi,\left.x(\cdot)\right|_{J} \in P C$ and

$$
\begin{aligned}
x(t)= & C(t) \varphi(0)+S(t)\left(z+g_{1}(0, \varphi, 0)\right)-\int_{0}^{t} C(t-s) g_{1}\left(s, x_{s}, \int_{0}^{s} k_{1}\left(s, r, x_{r}\right) d r\right) d s \\
& +\int_{0}^{t} S(t-s) g_{2}\left(s, x_{s}, \int_{0}^{s} k_{2}\left(s, r, x_{r}\right) d r\right) d s+\sum_{0<t_{i}<t} C\left(t-t_{i}\right) I_{i}^{1}\left(x_{t_{i}}\right) \\
& +\sum_{0<t_{i}<t} S\left(t-t_{i}\right) I_{i}^{2}\left(x_{t_{i}}\right), \quad t \in J .
\end{aligned}
$$

For system (1), we make the following hypotheses.

$\left(\mathrm{H}_{1}\right)$ The functions $g_{j}: J \times B \times X \rightarrow X(j=1,2)$ satisfy the following conditions:

(1) For every $(\phi, x) \in \beta \times X, g_{j}(\cdot, \phi, x)$ are strongly measurable and $g_{j}(t, \cdot, \cdot)$ are continuous for every $t \in J$;

(2) There are integrable functions $p_{j}: J \rightarrow \mathbb{R}^{+}(j=1,2)$ such that

$$
\left\|g_{j}(t, \phi, x)\right\| \leq p_{j}(t)\left(\|\phi\|_{\beta}+\|x\|\right), \quad t \in J, \phi \in ß, x \in X
$$

(3) For any bounded set $V \subset P C$, there are integrable functions $\gamma_{j}: J \rightarrow \mathbb{R}^{+}(j=1,2)$ such that

$$
\alpha\left(g_{j}\left(t, V_{t}, V(t)\right)\right) \leq \gamma_{j}(t)\left[\alpha\left(V_{t}\right)+\alpha(V(t))\right], \quad t \in J,
$$

where $V_{t}=\left\{x_{t}: x \in V\right\} \subset \beta(t \in J)$. 
$\left(\mathrm{H}_{2}\right) k_{j}: \Delta \times \beta \rightarrow X(j=1,2, \Delta=\{(t, s) \in J \times J: 0 \leq s \leq t \leq 1\})$ satisfies the following conditions:

(1) For every $\phi \in \beta, k_{j}(\cdot, \cdot, \phi)$ are strongly measurable and $k_{j}(t, s, \cdot)$ are continuous for every $(t, s) \in \Delta$;

(2) There are continuous functions $q_{j}: \Delta \rightarrow \mathbb{R}^{+}(j=1,2)$ such that

$$
\left\|k_{j}(t, s, \phi)\right\| \leq q_{j}(t, s)\|\phi\|_{\beta}, \quad(t, s) \in \Delta, \phi \in \beta
$$

(3) For any bounded set $V \subset P C$, there are continuous functions $\mu_{j}: \Delta \rightarrow \mathbb{R}^{+}(j=1,2)$ such that

$$
\alpha\left(k_{j}\left(t, s, V_{s}\right)\right) \leq \mu_{j}(t, s) \alpha\left(V_{s}\right), \quad(t, s) \in \Delta
$$

$\left(\mathrm{H}_{3}\right)$ The functions $I_{i}^{j}: B \rightarrow X(i=1, \ldots, n, j=1,2)$ are continuous and there are constants $c_{i}^{j} \geq 0, d_{i}^{j}>0$ such that

$$
\left\|I_{i}^{j}(\phi)\right\| \leq c_{i}^{j}\|\phi\|_{\beta}+d_{i}^{j}, \quad \phi \in \beta
$$

Let the function $y:(-\infty, b] \rightarrow X$ be defined by $y_{0}=\varphi$ and

$$
y(t)=C(t) \varphi(0)+S(t)\left(z+g_{1}(0, \varphi, 0)\right), \quad t \in J
$$

Theorem 3.2 Suppose that the cosine function family $C(t), t \in \mathbb{R}$, is equicontinuous, $g_{1}$, $g_{2}$ satisfy the condition $\left(\mathrm{H}_{1}\right),\left(\mathrm{H}_{2}\right)$ and $\left(\mathrm{H}_{3}\right)$ are satisfied. Then the impulsive second-order system (1) has at least one mild solution.

Proof Let $S(b)$ be the space $S(b)=\left\{x:(-\infty, b] \rightarrow X, x_{0}=0,\left.x\right|_{J} \in P C\right\}$ endowed with the supremum norm $\|\cdot\|_{b}$. The map $F: S(b) \rightarrow S(b)$ is defined by

$$
(F x)(t)=\left\{\begin{array}{l}
0, \quad t \leq 0, \\
-\int_{0}^{t} C(t-s) g_{1}\left(s, x_{s}+y_{s}, \int_{0}^{s} k_{1}\left(s, r, x_{r}+y_{r}\right) d r\right) d s \\
\quad+\int_{0}^{t} S(t-s) g_{2}\left(s, x_{s}+y_{s}, \int_{0}^{s} k_{2}\left(s, r, x_{r}+y_{r}\right) d r\right) d s \\
\quad+\sum_{0<t_{i}<t} C\left(t-t_{i}\right) I_{i}^{1}\left(x_{t_{i}}+y_{t_{i}}\right) \\
\quad+\sum_{0<t_{i}<t} S\left(t-t_{i}\right) I_{i}^{2}\left(x_{t_{i}}+y_{t_{i}}\right), \quad t \in J .
\end{array}\right.
$$

Clearly, $\left\|x_{t}+y_{t}\right\|_{\beta} \leq\left\|x_{t}\right\|_{\beta}+\sup _{t \in J}\left\|y_{t}\right\|_{\beta} \leq K_{b}\|x\|_{t}+M$, where $K_{b}=\sup _{0 \leq t \leq b} K(t), M=$ $\sup _{t \in J}\left\|y_{t}\right\|_{B},\|x\|_{t}=\sup _{0 \leq s \leq t}\|x(s)\|$. Thus $F$ is well defined with values in $S(b)$. In addition, from the axioms of phase space, the Lebesgue dominated convergence theorem and the conditions $\left(\mathrm{H}_{1}\right),\left(\mathrm{H}_{2}\right)$ and $\left(\mathrm{H}_{3}\right)$, we can show that $F$ is continuous (see [5]). It is easy to see that if $x$ is a fixed point of $F$, then $x+y$ is a mild solution of system (1).

Firstly, we show that the set

$$
\Omega_{0}=\{x \in S(b): x=\lambda F x \text { for some } \lambda \in(0,1)\}
$$

is bounded. In fact, if $x \in \Omega_{0}$, then there exists a $\lambda \in(0,1)$ such that $x=\lambda F x$. 
When $t \in J_{0}=\left[0, t_{1}\right]$, notice that $\left\|x_{t}\right\|_{B} \leq K_{b}\|x\|_{t}$ and $\|x\|_{t}$ is continuous nondecreasing on $J_{0}$. We have, by $(8)$ and $\left(\mathrm{H}_{1}\right)$,

$$
\begin{aligned}
\|x(t)\| \leq & N \int_{0}^{t} p_{1}(s)\left(\left\|x_{s}+y_{s}\right\|_{B}+\int_{0}^{s} q_{1}(s, r)\left\|x_{r}+y_{r}\right\|_{B} d r\right) d s \\
& +\bar{N} \int_{0}^{t} p_{2}(s)\left(\left\|x_{s}+y_{s}\right\|_{B}+\int_{0}^{s} q_{2}(s, r)\left\|x_{r}+y_{r}\right\|_{\beta} d r\right) d s \\
\leq & N M \int_{0}^{t_{1}}\left(p_{1}(s)+\int_{0}^{s} q_{1}(s, r) d r\right) d s \\
& +N K_{b} \int_{0}^{t}\left(p_{1}(s)+\int_{0}^{s} q_{1}(s, r) d r\right)\|x\|_{s} d s \\
& +\bar{N} M \int_{0}^{t_{1}}\left(p_{2}(s)+\int_{0}^{s} q_{2}(s, r) d r\right) d s \\
& +\bar{N} K_{b} \int_{0}^{t}\left(p_{2}(s)+\int_{0}^{s} q_{2}(s, r) d r\right)\|x\|_{s} d s \\
\leq & (\bar{N}+N) M \int_{0}^{t_{1}}\left(p_{1}(s)+p_{2}(s)+\int_{0}^{s} q_{1}(s, r) d r+\int_{0}^{s} q_{2}(s, r) d r\right) d s \\
& +(\bar{N}+N) K_{b} \int_{0}^{t}\left(p_{1}(s)+p_{2}(s)+\int_{0}^{s} q_{1}(s, r) d r+\int_{0}^{s} q_{2}(s, r) d r\right)\|x\|_{s} d s .
\end{aligned}
$$

Consequently,

$$
\begin{aligned}
\|x\|_{t} \leq & (\bar{N}+N) M \int_{0}^{t_{1}}\left(p_{1}(s)+p_{2}(s)+\int_{0}^{s} q_{1}(s, r) d r+\int_{0}^{s} q_{2}(s, r) d r\right) d s \\
& +(\bar{N}+N) K_{b} \int_{0}^{t}\left(p_{1}(s)+p_{2}(s)+\int_{0}^{s} q_{1}(s, r) d r+\int_{0}^{s} q_{2}(s, r) d r\right)\|x\|_{s} d s .
\end{aligned}
$$

By well-known Gronwall's lemma and (10), there are constants $G_{0}>0$ independent of $x$ and $\lambda \in(0,1)$ such that $\|x(t)\| \leq G_{0}$ and $\left\|x_{t}\right\|_{\beta} \leq K_{b} G_{0}, t \in J_{0}$. It follows from this and the condition $\left(\mathrm{H}_{3}\right)$ that

$$
\begin{aligned}
& \left\|I_{1}^{j}\left(x_{t_{1}}+y_{t_{1}}\right)\right\| \leq c_{1}^{j}\left(K_{b} G_{0}+M\right)+d_{1}^{j}=\delta_{j} \quad(j=1,2), \\
& \left\|x\left(t_{1}^{+}\right)\right\|=\left\|x\left(t_{1}\right)+I_{1}^{1}\left(x_{t_{1}}+y_{t_{1}}\right)\right\| \leq G_{0}+\delta_{1} .
\end{aligned}
$$

Nextly, when $t \in J_{1}=\left(t_{1}, t_{2}\right]$, let

$$
u(t)= \begin{cases}x(t), & t \in\left(t_{1}, t_{2}\right], \\ x\left(t_{1}^{+}\right), & t=t_{1} .\end{cases}
$$

Then $u \in C\left(\left[t_{1}, t_{2}\right], X\right)$. Similar to (10), we get

$$
\begin{aligned}
\|u(t)\| \leq & (\bar{N}+N) M \int_{0}^{t_{2}}\left(p_{1}(s)+p_{2}(s)+\int_{0}^{s} q_{1}(s, r) d r+\int_{0}^{s} q_{2}(s, r) d r\right) d s \\
& +(\bar{N}+N) K_{b} G_{0} \int_{0}^{t_{1}}\left(p_{1}(s)+p_{2}(s)+\int_{0}^{s} q_{1}(s, r) d r+\int_{0}^{s} q_{2}(s, r) d r\right) d s
\end{aligned}
$$




$$
\begin{aligned}
& +(\bar{N}+N) K_{b} \int_{t_{1}}^{t}\left(p_{1}(s)+p_{2}(s)+\int_{0}^{s} q_{1}(s, r) d r+\int_{0}^{s} q_{2}(s, r) d r\right)\|u\|_{s} d s \\
& +N\left\|I_{1}^{1}\left(x_{t_{1}}+y_{t_{1}}\right)\right\|+\bar{N}\left\|I_{1}^{2}\left(x_{t_{1}}+y_{t_{1}}\right)\right\|
\end{aligned}
$$

where $\|x\|_{t} \leq \sup _{0 \leq s \leq t_{1}}\|x(s)\|+\sup _{t_{1} \leq s \leq t}\|u(s)\|=:\|x\|_{t_{1}}+v(t)$. Equation (11) implies that

$$
\begin{aligned}
v(t) \leq & N \delta_{1}+\bar{N} \delta_{2}+(\bar{N}+N)\left(M+K_{b} G_{0}\right) \int_{0}^{t_{2}} a(s) d s \\
& +(\bar{N}+N) K_{b} \int_{t_{1}}^{t} a(s) v(s) d s, \quad t \in\left[t_{1}, t_{2}\right]
\end{aligned}
$$

where $a(s)=p_{1}(s)+p_{2}(s)+\int_{0}^{s} q_{1}(s, r) d r+\int_{0}^{s} q_{2}(s, r) d r$. Using Gronwall's lemma once again and (12), there is a constant $G_{1}>0$ independent of $v$ and $\lambda \in(0,1)$ such that $v(t) \leq G_{1}$, $t \in\left[t_{1}, t_{2}\right]$. Thence $\|x(t)\| \leq G_{1}$ and $\left\|x_{t}\right\|_{\beta} \leq K_{b}\left(G_{0}+G_{1}\right)$ for $t \in J_{1}$.

It is similar to the proof above, there is a constant $G_{i}>0$ independent of $x$ and $\lambda \in(0,1)$ such that $\|x(t)\| \leq G_{i}, t \in J_{i}(i=2,3, \ldots, n)$. Let $G=\max \left\{G_{0}, G_{1}, \ldots, G_{n}\right\}$, then $\|x(t)\| \leq G$, $t \in J$, i.e., $\Omega_{0}$ is bounded.

Lastly, we verify that all the conditions of Lemma 2.6 are satisfied. Let $R>G$ and

$$
\Omega_{R}=\left\{x \in S(b):\|x\|_{b}<R\right\} .
$$

Then $\Omega_{R}$ is a bounded open set and $0 \in \Omega$. Since $R>G$, we know that $x \neq \lambda F x$ for any $x \in \partial \Omega_{R}$ and $\lambda \in(0,1)$.

Nextly, let $V \subset \bar{\Omega}_{R}$ be a countable set and $V \subset \overline{c o}(\{0\} \cup F(V))$. Then

$$
V(t) \subset \overline{c o}(\{0\} \cup F(V)(t)), \quad t \in[0, b] .
$$

It follows from $\left(\mathrm{H}_{1}\right)-\left(\mathrm{H}_{3}\right)$ and Lemma 2.2 that $F(V)$ is equicontinuous on every interval $\bar{J}_{i}=\left[t_{i}, t_{i+1}\right](i=0,1, \ldots, n)$, which together with (13) implies that $V$ is equicontinuous on every $\bar{J}_{i}(i=0,1, \ldots, n)$.

When $t \in J_{0}=\left[0, t_{1}\right]$, by the property of noncompactness measure, $\left(\mathrm{H}_{1}\right)(3),\left(\mathrm{H}_{2}\right)(3)$ and Lemma 2.5, we have

$$
\begin{aligned}
\alpha(V(t)) \leq & \alpha(F(V)(t)) \\
\leq & 2 N \int_{0}^{t} \alpha\left(g_{1}\left(s, V_{s}+y_{s}, \int_{0}^{s} k_{1}\left(s, r, V_{r}+y_{r}\right) d r\right)\right) d s \\
& +2 \bar{N} \int_{0}^{t} \alpha\left(g_{2}\left(s, V_{s}+y_{s}, \int_{0}^{s} k_{2}\left(s, r, V_{r}+y_{r}\right) d r\right)\right) d s \\
\leq & 2(\bar{N}+N) \int_{0}^{t}\left[\left(\gamma_{1}(s)+\gamma_{2}(s)\right) \alpha\left(V_{s}\right)+2 \int_{0}^{s}\left(\mu_{1}(s, r)+\mu_{2}(s, r)\right) \alpha\left(V_{r}\right) d r\right] d s \\
\leq & 2(\bar{N}+N) K_{b} \int_{0}^{t}\left[\sum_{k=1}^{2} \gamma_{k}(s)+2 \int_{0}^{s} \sum_{i=1}^{2} \mu_{k}(s, r) d r\right] \sup _{0 \leq \tau \leq s} \alpha(V(\tau)) d s,
\end{aligned}
$$


where $\alpha\left(V_{t}\right) \leq \sup _{0 \leq s \leq t} \alpha(V(s))$. Let $m(t)=\sup _{0 \leq s \leq t} \alpha(V(s)), t \in J_{0}$. Lemma 2.3 implies that $m \in C\left(J_{0}, \mathbb{R}^{+}\right)$and

$$
m(t) \leq 2(\bar{N}+N) K_{b} \int_{0}^{t}\left[\gamma_{1}(s)+\gamma_{2}(s)+2 \int_{0}^{s}\left(\mu_{1}(s, r)+\mu_{2}(s, r)\right) d r\right] m(s) d s, \quad t \in J_{0} .
$$

From this and Gronwall's lemma, we know that $m(t)=0$ and $\alpha(V(t))=0, t \in J_{0}$. Therefore $V$ is a relative compact set in $C\left(J_{0}, X\right)$. Since

$$
0 \leq \alpha\left(V_{t_{1}}\right) \leq \alpha_{c}(V)=\sup _{0 \leq t \leq t_{1}} \alpha(V(t))=0
$$

and $I_{1}^{j}(\cdot)$ is continuous, $\alpha\left(V_{t_{1}}+y_{t_{1}}\right) \leq \alpha\left(V_{t_{1}}\right)=0, \alpha\left(I_{1}^{j}\left(V_{t_{1}}+y_{t_{1}}\right)\right)=0(j=1,2)$.

When $t \in \bar{J}_{1}=\left[t_{1}, t_{2}\right]$, similar to (14), it is easy to get

$$
\begin{aligned}
\alpha(V(t)) \leq & 2(\bar{N}+N) K_{b} \int_{0}^{t} \gamma(s) \sup _{0 \leq \tau \leq s} \alpha(V(\tau)) d s \\
& +N \alpha\left(I_{1}^{1}\left(V_{t_{1}}+y_{t_{1}}\right)\right)+\bar{N} \alpha\left(I_{1}^{2}\left(V_{t_{1}}+y_{t_{1}}\right)\right) \\
\leq & 2(\bar{N}+N) K_{b} \int_{t_{1}}^{t} \gamma(s) \sup _{t_{1} \leq \tau \leq s} \alpha(V(\tau)) d s, \quad t \in \bar{J}_{1},
\end{aligned}
$$

where $\gamma(s)=\gamma_{1}(s)+\gamma_{2}(s)+2 \int_{0}^{s}\left(\mu_{1}(s, r)+\mu_{2}(s, r)\right) d r$. Let $q(t)=\sup _{t_{1} \leq s \leq t} \alpha(V(s)), t \in \bar{J}_{1}$. Equation (15) implies that

$$
q(t) \leq 2(\bar{N}+N) \int_{t_{1}}^{t} \gamma(s) q(s) d s, \quad t \in \bar{J}_{1}
$$

Therefore $\alpha(V(t))=0, t \in \bar{J}_{1}$ and $V$ is a relative compact set in $C\left(\bar{J}_{1}, X\right)$.

Similarly, we can show that $V$ is a relative compact set in $C\left(\bar{J}_{i}, X\right)(i=2,3, \ldots, n)$, so $V$ is a relative compact set in $S(b)$. Lemma 2.6 concludes that $F$ has a fixed point in $\bar{\Omega}_{R}$. Let $x$ be a fixed point of $F$ on $S(b)$. Then $z=x+y$ is a mild solution of system (1).

Nextly, we discuss the existence of mild solutions for the impulsive system (2).

Definition 3.3 A function $x:(-\infty, b] \rightarrow X$ is said to be a mild solution of system (2) if $x_{0}=\phi, x_{0}^{\prime}=\psi,\left.x(\cdot)\right|_{J} \in P C^{1}$ and

$$
\begin{aligned}
x(t)= & C(t) \phi(0)+S(t)(\psi(0)+g(0, \phi, \psi))-\int_{0}^{t} C(t-s) g\left(s, x_{s}, x_{s}^{\prime}\right) d s \\
& +\int_{0}^{t} S(t-s) \int_{0}^{s} f\left(r, x_{r}, x_{r}^{\prime}\right) d r d s+\sum_{0<t_{i}<t} C\left(t-t_{i}\right) I_{i}^{1}\left(x_{t_{i}}, x_{t_{i}}^{\prime}\right) \\
& +\sum_{0<t_{i}<t} S\left(t-t_{i}\right) I_{i}^{2}\left(x_{t_{i}}, x_{t_{i}}^{\prime}\right), \quad t \in J .
\end{aligned}
$$


Differentiate (16) to get

$$
\begin{aligned}
x^{\prime}(t)= & A S(t) \phi(0)+C(t)(\psi(0)+g(0, \phi, \psi))-g\left(t, x_{t}, x_{t}^{\prime}\right) \\
& -\int_{0}^{t} A S(t-s) g\left(s, x_{s}, x_{s}^{\prime}\right) d s+\int_{0}^{t} C(t-s) \int_{0}^{s} f\left(r, x_{r}, x_{r}^{\prime}\right) d r d s \\
& +\sum_{0<t_{i}<t} A S\left(t-t_{i}\right) I_{i}^{1}\left(x_{t_{i}}, x_{t_{i}}^{\prime}\right)+\sum_{0<t_{i}<t} C\left(t-t_{i}\right) I_{i}^{2}\left(x_{t_{i}}, x_{t_{i}}^{\prime}\right), \quad t \in J .
\end{aligned}
$$

Let functions $y, y^{\prime}:(-\infty, b] \rightarrow X$ be defined by $y_{0}=\varphi, y_{0}^{\prime}=\psi$ and

$$
y(t)=C(t) \varphi(0)+S(t) \psi(0), \quad y^{\prime}(t)=A S(t) \varphi(0)+C(t) \psi(0), \quad t \in J .
$$

Clearly,

$$
\left\|y_{t}\right\|_{\beta} \leq K_{b}\|y\|_{b}+M_{b}\|\varphi\|_{\beta}=\bar{M}, \quad\left\|y_{t}^{\prime}\right\|_{\beta} \leq K_{b}\left\|y^{\prime}\right\|_{b}+M_{b}\|\psi\|_{\beta}=M^{\prime},
$$

where $\|y\|_{b}=\sup _{0 \leq t \leq b}\|y(t)\|,\left\|y^{\prime}\right\|_{b}=\sup _{0 \leq t \leq b}\left\|y^{\prime}(t)\right\|$.

Let $S^{1}(b)$ be the space $S^{1}(b)=\left\{x:(-\infty, b] \rightarrow X: x_{0}=0, x_{0}^{\prime}=0,\left.x(\cdot)\right|_{J} \in P C^{1}\right\}$ endowed with the supremum norm $\|\cdot\|_{1 b}$.

We make the following hypotheses for convenience.

$\left(\mathrm{H}_{f}\right) f: J \times B \times B \rightarrow X$ satisfies the following conditions:

(1) For every $x \in S^{1}(b)$, the function $t \rightarrow f\left(t, x_{t}, x_{t}^{\prime}\right)$ is strongly measurable and $f(t, \cdot, \cdot)$ is continuous for every $t \in J$;

(2) There is an integrable function $p: J \rightarrow \mathbb{R}^{+}$such that

$$
\|f(t, u, v)\| \leq p(t)\left(\|u\|_{\beta}+\|v\|_{\beta}\right), \quad t \in J, u, v \in \beta
$$

(3) For any bounded set $V \subset P C^{1}$, there is an integrable function $\mu: J \rightarrow \mathbb{R}^{+}$such that

$$
\alpha\left(f\left(t, V_{t}, V_{t}^{\prime}\right)\right) \leq \mu(t)\left(\alpha\left(V_{t}\right)+\alpha\left(V_{t}^{\prime}\right)\right), \quad t \in J
$$

where $V_{t}=\left\{x_{t}: x \in V\right\}, V_{t}^{\prime}=\left\{x_{t}^{\prime}: x^{\prime} \in V^{\prime}\right\} \subset \beta(t \in J), V^{\prime} \subset P C$.

$\left(\mathrm{H}_{g}\right) g: J \times B \times B \rightarrow E$ satisfies the following conditions:

(1) The function $g(\cdot)$ is continuous, there are constants $c>0, d \geq 0$ such that $c K_{b}<1$ and

$$
\|g(t, u, v)\|_{E} \leq c\left(\|u\|_{\beta}+\|v\|_{\beta}\right)+d, \quad t \in J, u, v \in \beta
$$

(2) For every bounded set $Q \subset S^{1}(b)$, the set of functions $\left\{\left(\widetilde{\omega_{x}}\right)_{i}(t): x \in Q\right\}$ is uniformly equicontinuous on $\bar{J}_{i}=\left[t_{i}, t_{i+1}\right]$ for every $i=0,1, \ldots, n$, where $\omega_{x}(t)=g\left(t, x_{t}, x_{t}^{\prime}\right)$;

(3) For any bounded set $V \subset P C^{1}, \alpha\left(g\left(t, V_{t}, V_{t}^{\prime}\right)\right) \leq c\left(\alpha\left(V_{t}\right)+\alpha\left(V_{t}^{\prime}\right)\right), t \in J$.

$\left(\mathrm{H}_{I}\right)$ The functions $I_{i}^{1}: \beta \times \beta \rightarrow E, I_{i}^{2}: \beta \times \beta \rightarrow X(i=1, \ldots, n)$ are continuous and there are constants $c_{i}^{j} \geq 0, d_{i}^{j} \geq 0$ such that

$$
\left\|I_{i}^{1}(u, v)\right\|_{E} \leq c_{i}^{1}\left(\|u\|_{\beta}+\|v\|_{\beta}\right)+d_{i}^{1}, \quad\left\|I_{i}^{2}(u, v)\right\| \leq c_{i}^{2}\left(\|u\|_{\beta}+\|v\|_{\beta}\right)+d_{i}^{2}, \quad u, v \in \beta .
$$

Theorem 3.4 Let the conditions $\left(\mathrm{H}_{f}\right),\left(\mathrm{H}_{g}\right)$ and $\left(\mathrm{H}_{I}\right)$ be satisfied, the cosine function family $C(t), t \in \mathbb{R}$, be equicontinuous and $\varphi(0) \in E$. Then system (2) has at least one mild solution. 
Proof Let the function $z:(-\infty, 0] \rightarrow X$ be defined by $z_{0}=x_{0}^{\prime}, z(t)=x^{\prime}(t), t \in J$, the map $\Gamma=\left(\Gamma_{1}, \Gamma_{2}\right): S(b) \times S(b) \rightarrow S(b)$ be defined by

$$
\Gamma_{1}(x, z)(t)=\left\{\begin{array}{l}
0, \quad t \leq 0, \\
S(t) g(0, \varphi, \psi)-\int_{0}^{t} C(t-s) g\left(s, x_{s}+y_{s}, z_{s}+y_{s}^{\prime}\right) d s \\
\quad+\int_{0}^{t} S(t-s) \int_{0}^{s} f\left(r, x_{r}, x_{r}^{\prime}\right) d r d s \\
\quad+\sum_{0<t_{i}<t} C\left(t-t_{i}\right) I_{i}^{1}\left(x_{t_{i}}+y_{t_{i}}, z_{t_{i}}+y_{t_{i}}^{\prime}\right) \\
\quad+\sum_{0<t_{i}<t} S\left(t-t_{i}\right) I_{i}^{2}\left(x_{t_{i}}+y_{t_{i}}, z_{t_{i}}+y_{t_{i}}^{\prime}\right), \quad t \in J,
\end{array}\right.
$$

and $\Gamma_{1}(x, z)^{\prime}(t)=\Gamma_{2}(x, z)(t)$,

$$
\Gamma_{2}(x, z)(t)=\left\{\begin{array}{l}
0, \quad t \leq 0, \\
C(t) g(0, \varphi, \psi)-g\left(t, x_{t}+y_{t}, z_{t}+y_{t}^{\prime}\right) \\
\quad-\int_{0}^{t} A S(t-s) g\left(s, x_{s}+y_{s}, z_{s}+y_{s}^{\prime}\right) d s \\
\quad+\int_{0}^{t} C(t-s) \int_{0}^{s} f\left(r, x_{r}, x_{r}^{\prime}\right) d r d s \\
\quad+\sum_{0<t_{i}<t} A S\left(t-t_{i}\right) I_{i}^{1}\left(x_{t_{i}}+y_{t_{i}}, z_{t_{i}}+y_{t_{t}}^{\prime}\right) \\
\quad+\sum_{0<t_{i}<t} C\left(t-t_{i}\right) I_{i}^{2}\left(x_{t_{i}}+y_{t_{i}}, z_{t_{i}}+y_{t_{i}}^{\prime}\right), \quad t \in J .
\end{array}\right.
$$

The product space $S(b) \times S(b)$ is endowed with the norm $\|(x, z)\|_{b}=\|x\|_{b}+\|z\|_{b}$. Then $\Gamma_{1}$, $\Gamma_{2}$ are well defined and with values in $S(b)$. In addition, from the axioms of phase space, the Lebesgue dominated convergence theorem and the conditions $\left(\mathrm{H}_{f}\right),\left(\mathrm{H}_{g}\right)$ and $\left(\mathrm{H}_{I}\right)$, we can show that $\Gamma=\left(\Gamma_{1}, \Gamma_{2}\right)$ is continuous. It is easy to see that if $(x, z)$ is a fixed point of $\Gamma$, then $x+y$ is a mild solution of system (2).

Firstly, we show that the set

$$
\Omega_{0}=\{(x, z) \in S(b) \times S(b):(x, z)=\lambda \Gamma(x, z) \text { for some } \lambda \in(0,1)\}
$$

is bounded. If $x \in \Omega_{0}$, there exists a $\lambda \in(0,1)$ such that $x=\lambda \Gamma_{1}(x, z)$ and $z=\lambda \Gamma_{2}(x, z)$.

When $t \in J_{0}=\left[0, t_{1}\right]$, it follows from (18), (19) and $\left(\mathrm{H}_{f}\right)(2),\left(\mathrm{H}_{g}\right)(1),\left(\mathrm{H}_{I}\right)$ that

$$
\begin{aligned}
\|x(t)\| \leq & \left\|\Gamma_{1}(x, z)(t)\right\| \leq \bar{N}\|g(0, \varphi, \psi)\|_{E} \\
& +(N+\bar{N}) \int_{0}^{t}\left(\left\|g\left(s, x_{s}+y_{s}, z_{s}+y_{s}^{\prime}\right)\right\|+\int_{0}^{s}\left\|f\left(r, x_{r}+y_{r}, z_{r}+y_{r}^{\prime}\right)\right\| d r\right) d s \\
\leq & \bar{N}\|g(0, \varphi, \psi)\|_{E}+N \int_{0}^{t}\left[c\left(\left\|x_{s}\right\|_{\beta}+\left\|z_{s}\right\|_{\beta}+\bar{M}+M^{\prime}\right)+d\right] d s \\
& +\bar{N} \int_{0}^{t} \int_{0}^{s} p(r)\left(\left\|x_{r}\right\|_{B}+\left\|z_{r}\right\|_{B}+\bar{M}+M^{\prime}\right) d r d s \\
\leq & \bar{N}\|g(0, \varphi, \psi)\|_{E}+\left(\bar{M}+M^{\prime}\right) \int_{0}^{t_{1}}\left(N c+\bar{N} \int_{0}^{s} p(r) d r\right) d s+N b d \\
& +(N c+\bar{N}) K_{b} \int_{0}^{t}\left(1+\int_{0}^{s} p(r) d r\right)\left(\|x\|_{s}+\|z\|_{s}\right) d s,
\end{aligned}
$$




$$
\begin{aligned}
\|z(t)\| \leq & \left\|\Gamma_{2}(x, z)(t)\right\| \leq N\|g(0, \varphi, \psi)\|_{E}+c\left(\left\|x_{t}+y_{t}\right\|_{B}+\left\|z_{t}+y_{t}^{\prime}\right\|_{B}\right)+d \\
& +\left(\bar{M}+M^{\prime}\right) \int_{0}^{t_{1}}\left(N_{1} c+N \int_{0}^{s} p(r) d r\right) d s+N_{1} b d \\
& +\left(N_{1} c+N\right) K_{b} \int_{0}^{t}\left(1+\int_{0}^{s} p(r) d r\right)\left(\|x\|_{s}+\|z\|_{s}\right) d s \\
\leq & N\|g(0, \varphi, \psi)\|_{E}+\left(\bar{M}+M^{\prime}\right)\left[c+\int_{0}^{t_{1}}\left(N_{1} c+N \int_{0}^{s} p(r) d r\right) d s\right] \\
& +\left(N_{1} b+1\right) d+c K_{b}\left(\|x\|_{t}+\|z\|_{t}\right) \\
& +\left(N_{1} c+N\right) K_{b} \int_{0}^{t}\left(1+\int_{0}^{s} p(r) d r\right)\left(\|x\|_{s}+\|z\|_{s}\right) d s .
\end{aligned}
$$

Equations (20) and (21) imply that

$$
\begin{aligned}
\|x\|_{t}+\|z\|_{t} \leq & \frac{1}{1-c K_{b}}\left[(N+\bar{N})\|g(0, \varphi, \psi)\|_{E}+\left(N b+N_{1} b+1\right) d\right. \\
& \left.+\left(\bar{M}+M^{\prime}\right)\left(c+\left(N+N_{1}\right) c t_{1}+(N+\bar{N}) \int_{0}^{t_{1}} \int_{0}^{s} p(r) d r d s\right)\right] \\
& +\frac{\left(N c+\bar{N}+N_{1} c+N\right) K_{b}}{1-c K_{b}} \int_{0}^{t}\left(1+\int_{0}^{s} p(r) d r\right)\left(\|x\|_{s}+\|z\|_{s}\right) d s .
\end{aligned}
$$

Since $\|x\|_{t}+\|z\|_{t} \in C\left(J_{0}, X\right)$, by Gronwall's lemma and (22), there is a constant $G_{0}>0$ such that $\|x\|_{t}+\|z\|_{t} \leq G_{0}, t \in J_{0}$. Therefore $\|x(t)\|+\|z(t)\| \leq G_{0}, t \in J_{0}$ and $\left\|x_{t}\right\|_{\beta} \leq K_{b} G_{0}$, $\left\|z_{t}\right\|_{B} \leq K_{b} G_{0}, t \in J_{0}$. It follows from this and the condition $\left(\mathrm{H}_{I}\right)$ that

$$
\begin{aligned}
& \left\|I_{1}^{j}\left(x_{t_{1}}+y_{t_{1}}, z_{t_{1}}+y_{t_{1}}^{\prime}\right)\right\|_{E} \leq c_{1}^{j}\left(2 K_{b} G_{0}+\bar{M}+M^{\prime}\right)+d_{1}^{j}=: \eta_{j} \quad(j=1,2), \\
& \left\|x\left(t_{1}^{+}\right)\right\|=\left\|x\left(t_{1}\right)+I_{1}^{1}\left(x_{t_{1}}+y_{t_{1}}, z_{t_{1}}+y_{t_{1}}^{\prime}\right)\right\| \leq G_{0}+\eta_{1} \\
& \left\|z\left(t_{1}^{+}\right)\right\|=\left\|z\left(t_{1}\right)+I_{1}^{2}\left(x_{t_{1}}+y_{t_{1}}, z_{t_{1}}+y_{t_{1}}^{\prime}\right)\right\| \leq G_{0}+\eta_{2} .
\end{aligned}
$$

Nextly, when $t \in J_{1}=\left(t_{1}, t_{2}\right]$, let

$$
u(t)=\left\{\begin{array}{ll}
x(t), & t \in\left(t_{1}, t_{2}\right], \\
x\left(t_{1}^{+}\right), & t=t_{1},
\end{array} \quad v(t)= \begin{cases}z(t), & t \in\left(t_{1}, t_{2}\right] \\
z\left(t_{1}^{+}\right), & t=t_{1} .\end{cases}\right.
$$

Then $u, v \in C\left(\left[t_{1}, t_{2}\right], X\right)$. Similar to (20) and (21), we get

$$
\begin{aligned}
\|u(t)\| \leq & \bar{N}\|g(0, \varphi, \psi)\|_{E}+(N+\bar{N}) \int_{0}^{t}\left(c+\int_{0}^{s} p(r) d r\right)\left(\|x\|_{s}+\|z\|_{s}+M+M^{\prime}\right) d s \\
& +N b d+N\left\|I_{1}^{1}\left(x_{t_{1}}+y_{t_{1}}, z_{t_{1}}+y_{t_{1}}^{\prime}\right)\right\|_{E}+\bar{N}\left\|I_{1}^{2}\left(x_{t_{1}}+y_{t_{1}}, z_{t_{1}}+y_{t_{1}}^{\prime}\right)\right\| \\
\leq & \bar{N}\|g(0, \varphi, \psi)\|_{E}+(N+\bar{N})\left[\left(2 K_{b} G_{0}+\bar{M}+M^{\prime}\right) \int_{0}^{t_{1}}\left(c+\int_{0}^{s} p(r) d r\right) d s\right. \\
& \left.+N b d+\left(2 K_{b} G_{0}+\bar{M}+M^{\prime}\right) \int_{t_{1}}^{t_{2}}\left(c+\int_{0}^{s} p(r) d r\right) d s\right]+N \eta_{1}+\bar{N} \eta_{2} \\
& +(N+\bar{N}) K_{b} \int_{t_{1}}^{t}\left(c+\int_{0}^{s} p(r) d r\right)\left(\sup _{t_{1} \leq \tau \leq s}\|u(\tau)\|+\sup _{t_{1} \leq \tau \leq s}\|v(\tau)\|\right) d s,
\end{aligned}
$$




$$
\begin{aligned}
\|v(t)\| \leq & N\|g(0, \varphi, \psi)\|_{E}+c\left(\left\|x_{t}+y_{t}\right\|_{\beta}+\left\|z_{t}+y_{t}^{\prime}\right\|_{\beta}\right)+d+N_{1} \eta_{1}+N \eta_{2} \\
& +N_{1} b d+\left(N+N_{1}\right) \int_{0}^{t}\left(c+\int_{0}^{s} p(r) d r\right)\left(K_{b}\|x\|_{s}+K_{b}\|z\|_{s}+\bar{M}+M^{\prime}\right) d s \\
\leq & N\|g(0, \varphi, \psi)\|_{E}+c\left(2 K_{b} G_{0}+\bar{M}+M^{\prime}\right)+N_{1} \eta_{1}+N \eta_{2}+\left(N_{1} b+1\right) d \\
& +\left(N+N_{1}\right)\left(2 K_{b} G_{0}+\bar{M}+M^{\prime}\right) \int_{0}^{t_{2}}\left(c+\int_{0}^{s} p(r) d r\right) d s \\
& +\left(N+N_{1}\right) K_{b} \int_{t_{1}}^{t}\left(c+\int_{0}^{s} p(r) d r\right)\left(\sup _{t_{1} \leq \tau \leq s}\|u(\tau)\|+\sup _{t_{1} \leq \tau \leq s}\|v(\tau)\|\right) d s \\
& +c K_{b}\left(\sup _{t_{1} \leq s \leq t}\|u(s)\|+\sup _{t_{1} \leq s \leq t}\|v(s)\|\right) .
\end{aligned}
$$

We have, by (23) and (24),

$$
\begin{aligned}
\sup _{t_{1} \leq s \leq t} & \|u(s)\|+\sup _{t_{1} \leq s \leq t}\|v(s)\| \\
\leq & \frac{e_{1}+e_{2}}{1-c K_{b}}+\frac{\left(2 N+\bar{N}+N_{1}\right) K_{b}}{1-c K_{b}} \\
& \cdot \int_{t_{1}}^{t}\left(c+\int_{0}^{s} p(r) d r\right)\left(\sup _{t_{1} \leq \tau \leq s}\|u(\tau)\|+\sup _{t_{1} \leq \tau \leq s}\|v(\tau)\|\right) d s, \quad t \in\left[t_{1}, t_{2}\right],
\end{aligned}
$$

where

$$
\begin{aligned}
e_{1}= & (N+\bar{N})\left[\|g(0, \varphi, \psi)\|_{E}+\eta_{1}+\eta_{2}+\left(2 K_{b} G_{0}+\bar{M}+M^{\prime}\right) \int_{0}^{t_{2}}\left(c+\int_{0}^{s} p(r) d r\right) d s\right], \\
e_{2}= & \left(2 K_{b} G_{0}+\bar{M}+M^{\prime}\right)\left[c+\left(N+N_{1}\right) \int_{0}^{t_{2}}\left(c+\int_{0}^{s} p(r) d r\right) d s\right] \\
& +N_{1}\left(b d+\eta_{1}\right)+N\left(b d+\eta_{2}\right) .
\end{aligned}
$$

Using Gronwall's lemma once again and (25), there is a constant $G_{1}>0$ such that $\|u(t)\|+$ $\|v(t)\| \leq G_{1}, t \in\left[t_{1}, t_{2}\right]$, and so $\|x(t)\|+\|z(t)\| \leq G_{1}, t \in J_{1}$.

It is similar to the proof above, there are constants $G_{i}>0$ such that $\|x(t)\|+\left\|x^{\prime}(t)\right\| \leq G_{i}$, $t \in J_{i}(i=2,3, \ldots, n)$. Let $G=\max \left\{G_{0}, G_{1}, \ldots, G_{n}\right\}$, then $\|(x, z)\|_{b} \leq G$ and $\Omega_{0}$ is bounded.

Let $R>G$ and

$$
\Omega_{R}=\left\{(x, z) \in S(b) \times S(b):\|(x, z)\|_{b}<R\right\} .
$$

Then $\Omega_{R}$ is a bounded open set and $(0,0) \in \Omega$. Since $R>G$, we know that $(x, z) \neq \lambda \Gamma(x, z)$ for any $(x, z) \in \partial \Omega_{R}$ and $\lambda \in(0,1)$.

Suppose that $V \subset \bar{\Omega}_{R}$ is a countable set and $V \subset \overline{c o}(\{(0,0)\} \cup \Gamma(V))$. Let

$$
V_{1}=\{x \in S(b): \exists z \in S(b),(x, z) \in V\}, \quad V_{2}=\{z \in S(b): \exists x \in S(b),(x, z) \in V\} .
$$

Then we have

$$
\begin{aligned}
& V \subset V_{1} \times V_{2} \subset \overline{c o}\left(\{0\} \cup \Gamma_{1}(V)\right) \times \overline{c o}\left(\{0\} \cup \Gamma_{2}(V)\right) \\
& \quad \subset \overline{c o}\left(\{0\} \cup \Gamma_{1}\left(V_{1} \times V_{2}\right)\right) \times \overline{c o}\left(\{0\} \cup \Gamma_{2}\left(V_{1} \times V_{2}\right)\right) .
\end{aligned}
$$


It follows from (18), (19) and $\left(\mathrm{H}_{g}\right)(2)$ that $\Gamma_{j}\left(\left(\widetilde{V}_{1}\right)_{i} \times\left(\widetilde{V}_{2}\right)_{i}\right)(j=1,2)$ are equicontinuous on every interval $\bar{J}_{i}(i=0,1, \ldots, n)$, which together with $(26)$ implies that $\left(\widetilde{V}_{k}\right)_{i}(k=1,2)$ are equicontinuous on every interval $\bar{J}_{i}$.

In the following, we verify that the set $V_{1}, V_{2}$ is relatively compact in $P C$. Without loss of generality, we do not distinguish $\left.V_{k}\right|_{j}$ and $\widetilde{V}_{i}$, where $\left.V_{k}\right|_{J_{i}}(k=1,2)$ is the restriction of $V_{k}$ on $J_{i}=\left(t_{i}, t_{i+1}\right]$.

When $t \in J_{0}=\left[0, t_{1}\right]$, by the condition $\left(\mathrm{H}_{f}\right)(3),\left(\mathrm{H}_{g}\right)(3)$ and Lemma 2.5, we have

$$
\begin{aligned}
\alpha\left(V_{1}(t)\right) \leq & \alpha\left(\Gamma_{1}\left(V_{1} \times V_{2}\right)(t)\right) \\
\leq & 2 N \int_{0}^{t} \alpha\left(g\left(s, V_{1 s}+y_{s}, V_{2 s}+y_{s}^{\prime}\right)\right) d s \\
& +2 \bar{N} \int_{0}^{t} \alpha\left(\int_{0}^{s} f\left(r, V_{1 r}+y_{r}, V_{2 r}+y_{r}^{\prime}\right) d r\right) d s \\
\leq & 2(N+\bar{N}) \int_{0}^{t}\left(c+2 \int_{0}^{s} \mu(r) d r\right)\left(\alpha\left(V_{1 s}+y_{s}\right)+\alpha\left(V_{2 s}+y_{s}^{\prime}\right)\right) d s \\
\leq & 2(\bar{N}+N) K_{b} \int_{0}^{t}\left(c+2 \int_{0}^{s} \mu(r) d r\right) \\
& \times\left(\sup _{0 \leq \tau \leq s} \alpha\left(V_{1}(\tau)\right)+\sup _{0 \leq \tau \leq s} \alpha\left(V_{2}(\tau)\right)\right) d s, \\
\alpha\left(V_{2}(t)\right) \leq & \alpha\left(\Gamma_{2}\left(V_{1} \times V_{2}\right)(t)\right) \leq \alpha\left(g\left(t, V_{1 t}+y_{t}, V_{2 t}+y_{t}^{\prime}\right)\right) \\
& +2\left(N+N_{1}\right) K_{b} \int_{0}^{t}\left(c+2 \int_{0}^{s} \mu(r) d r\right) \\
& \times\left(\sup _{0 \leq \tau \leq s} \alpha\left(V_{1}(\tau)\right)+\sup _{0 \leq \tau \leq s} \alpha\left(V_{2}(\tau)\right)\right) d s \\
\leq & c K_{b}\left(\sup _{0 \leq s \leq t} \alpha\left(V_{1}(s)\right)+\sup _{0 \leq s \leq t} \alpha\left(V_{2}(s)\right)\right) \\
& +2\left(N+N_{1}\right) K_{b} \int_{0}^{t}\left(c+2 \int_{0}^{s} \mu(r) d r\right) \\
& \times\left(\sup _{0 \leq \tau \leq s} \alpha\left(V_{1}(\tau)\right)+\sup _{0 \leq \tau \leq s} \alpha\left(V_{2}(\tau)\right)\right) d s .
\end{aligned}
$$

Since $m_{j}(t)=: \sup _{0 \leq s \leq t} \alpha\left(V_{j}(s)\right)(j=1,2)$ are continuous nondecreasing on $J_{0},(27)$ and (28) imply that

$$
m_{1}(t)+m_{2}(t) \leq \frac{2\left(2 N+\bar{N}+N_{1}\right) K_{b}}{1-c K_{b}} \int_{0}^{t}\left(c+\int_{0}^{s} \mu(r) d r\right)\left(m_{1}(s)+m_{2}(s)\right) d s
$$

By Gronwall's lemma and (29), we have $\alpha\left(V_{k}(t)\right)=0(k=1,2), t \in J_{0}$. Lemma $2.3 \mathrm{im}$ plies that $V_{k}(k=1,2)$ is relatively compact in $C\left(J_{0}, X\right)$. Note that $\alpha\left(V_{j t_{1}}+y_{t_{1}}\right) \leq \alpha\left(V_{j t_{1}}\right) \leq$ $K_{b} \sup _{0 \leq s \leq t_{1}} \alpha\left(V_{j}(s)\right)=0$ and $I_{1}^{j}(\cdot, \cdot)(j=1,2)$ is continuous, we have

$$
\alpha\left(I_{1}^{1}\left(V_{1 t_{1}}+y_{t_{1}}, V_{2 t_{1}}+y_{t_{1}}^{\prime}\right)\right)=\alpha\left(I_{1}^{2}\left(V_{1 t_{1}}+y_{t_{1}}, V_{2 t_{1}}+y_{t_{1}}^{\prime}\right)\right)=0 .
$$


When $t \in \bar{J}_{1}=\left[t_{1}, t_{2}\right]$, it is similar to (27) and (28), we get

$$
\begin{aligned}
\alpha\left(V_{1}(t)\right) \leq & 2(\bar{N}+N) K_{b} \int_{t_{1}}^{t}\left(c+2 \int_{0}^{s} \mu(r) d r\right) \\
& \times\left(\sup _{t_{1} \leq \tau \leq s} \alpha\left(V_{1}(\tau)\right)+\sup _{t_{1} \leq \tau \leq s} \alpha\left(V_{2}(\tau)\right)\right) d s, \\
\alpha\left(V_{2}(t)\right) \leq & 2\left(N+N_{1}\right) K_{b} \int_{t_{1}}^{t}\left(c+2 \int_{0}^{s} \mu(r) d r\right) \\
& \times\left(\sup _{t_{1} \leq \tau \leq s} \alpha\left(V_{1}(\tau)\right)+\sup _{t_{1} \leq \tau \leq s} \alpha\left(V_{2}(\tau)\right)\right) d s \\
& +c K_{b}\left[\sup _{t_{1} \leq s \leq t} \alpha\left(V_{1}(s)\right)+\sup _{t_{1} \leq s \leq t} \alpha\left(V_{2}(s)\right)\right] .
\end{aligned}
$$

Equations (30) and (31) imply that

$$
\begin{aligned}
& \sup _{t_{1} \leq s \leq t} \alpha\left(V_{1}(s)\right)+\sup _{t_{1} \leq s \leq t} \alpha\left(V_{2}(s)\right) \\
& \quad \leq \frac{2\left(2 N+\bar{N}+N_{1}\right) K_{b}}{1-c K_{b}} \int_{t_{1}}^{t}\left(c+2 \int_{0}^{s} \mu(r) d r\right)\left(\sup _{t_{1} \leq \tau \leq s} \alpha\left(V_{1}(\tau)\right)+\sup _{t_{1} \leq \tau \leq s} \alpha\left(V_{2}(\tau)\right)\right) d s .
\end{aligned}
$$

Consequently, $\alpha\left(V_{k}(t)\right)=0(k=1,2), t \in J_{1}$. Lemma 2.3 implies that $V_{k}(k=1,2)$ are relatively compact in $C\left(\bar{J}_{1}, X\right)$.

Similarly, we can show that $V_{k}(k=1,2)$ are relatively compact in $C\left(\bar{J}_{i}, X\right)(i=2,3, \ldots, n)$. So $V_{k}(k=1,2)$ are relatively compact in $S(b)$. In view of Lemma 2.6 , we conclude that $\Gamma$ has a fixed point in $\bar{\Omega}_{R}$. Let $(x, z)$ be a fixed point of $\Gamma$ on $S(b)$. Then $x+y$ is a mild solution of system (2).

Theorem 3.5 Let the conditions $\left(\mathrm{H}_{f}\right),\left(\mathrm{H}_{g}\right)(2)$ and $\left(\mathrm{H}_{I}\right)$ be satisfied, the cosine function family $C(t), t \in \mathbb{R}$, be equicontinuous and $\varphi(0) \in$ E. Furthermore, suppose that the following condition is satisfied:

$\left(\mathrm{H}_{g}\right)\left(1^{\prime}\right)$ The function $g(\cdot)$ is continuous and $g(t, \cdot)$ satisfies the Lipschitz condition, that is, there is a constant $c>0$ such that

$$
\left\|g\left(t, u_{1}, v_{1}\right)-g\left(t, u_{2}, v_{2}\right)\right\|_{E} \leq c\left(\left\|u_{1}-u_{2}\right\|_{\beta}+\left\|v_{1}-v_{2}\right\|_{\beta}\right), \quad t \in J, u_{j}, v_{j} \in \beta(j=1,2),
$$

and $c K_{b}<1$. Then system (2) has at least one mild solution.

Proof We have, by the condition $\left(\mathrm{H}_{g}\right)\left(1^{\prime}\right)$,

$$
\begin{aligned}
& \|g(t, u, v)\|_{E} \leq c\left(\|u\|_{B}+\|v\|_{B}\right)+\|g(t, 0,0)\|_{E}, \quad t \in J, u, v \in \beta(j=1,2), \\
& \alpha\left(g\left(t, V_{t}, V_{t}^{\prime}\right)\right) \leq c\left(\alpha\left(V_{t}\right)+\alpha\left(V_{t}^{\prime}\right)\right), \quad t \in J .
\end{aligned}
$$

The rest of the proof is similar to the proof of Theorem 3.4, we omit it.

Remark 3.6 The similar restrictive conditions (3), (4) and (6) were used in $[1,2,13,14]$ even if $c K_{b}<1$. 


\section{Examples}

Let $X=L^{2}([0, \pi])$ and let $A$ be the operator given by $A f=f^{\prime \prime}$ with the domain

$$
D(A)=\left\{f \in X: f, f^{\prime} \text { are absolutely continuous, } f^{\prime \prime} \in X, f(0)=f(\pi)=0\right\} .
$$

It is well known that $A$ is the infinitesimal generator of a strongly continuous cosine family $C(t), t \in \mathbb{R}$, on $X$. Moreover, $A$ has discrete spectrum, the eigenvalues are $-n^{2}, n \in \mathbb{N}$, with corresponding normalized eigenvectors $z_{n}(\xi):=\sqrt{\frac{2}{\pi}} \sin (n \xi)$, and the following properties hold:

(a) $\left\{z_{n}: n \in \mathbb{N}\right\}$ is an orthonormal basis of $X$.

(b) For $f \in X, C(t) f=\sum_{n=1}^{\infty} \cos (n t)\left(f ; z_{n}\right) z_{n}$. Moreover, it follows from this expression that $S(t) f=\sum_{n=1}^{\infty} \frac{\sin (n t)}{n}\left(f ; z_{n}\right) z_{n}$, that $S(t)$ is compact for $t>0$ and that $\|C(t)\|=1$ and $\|S(t)\|=1$ for every $t \in \mathbb{R}$. Additionally, we observe that the operators $C(2 k \pi)$, $k \in \mathbb{N}$, are not compact.

(c) If $\Phi$ denotes the group of translations on $X$ defined by $\Phi(t) x(\xi)=\widetilde{x}(\xi+t)$, where $\widetilde{x}$ is the extension of $x$ with period $2 \pi$, then $C(t)=\frac{1}{2}(\Phi(t)+\Phi(-t)) ; A=B^{2}$, where $B$ is the infinitesimal generator of the group $\Phi$ and $E=\left\{x \in H^{1}(0, \pi): x(0)=x(\pi)=0\right\}$; see [1] for details. In particular, we observe that the inclusion $i_{c}: E \rightarrow X$ is compact.

In the next application, $B$ should be the phase space $\beta=C_{0} \times L^{2}(\rho, X)$ in [1], where $\rho$ : $(-\infty, 0] \rightarrow \mathbb{R}$ is a positive Lebesgue integrable function. We can take $H=1, M(t)=\gamma(-t)^{\frac{1}{2}}$ and $K(t)=1+\left(\int_{-t}^{0} \rho(\theta) d \theta\right)^{\frac{1}{2}}$ for $t \geq 0$.

Example 4.1 Consider the partial neutral functional integro-differential system:

$$
\left\{\begin{array}{l}
\frac{\partial}{\partial t}\left(\frac{\partial}{\partial t} u(t, \xi)+\int_{-\infty}^{t} h(s-t) u(s, \xi) d s\right) \\
\quad=\frac{\partial^{2}}{\partial \xi^{2}} u(t, \xi)+\int_{-\infty}^{t} F(t, s-t, \xi, u(s, \xi)) d s, \\
u(t, 0)=u(t, \pi)=0, \quad t \in[0, b], \\
u(\theta, \xi)=\varphi(\theta, \xi), \quad \theta \in(-\infty, 0], \xi \in[0, \pi], \\
\frac{\partial}{\partial t} u(0, \xi)=z(\xi), \quad \xi \in[0, \pi], \\
\Delta u\left(t_{i}\right)=\int_{-\infty}^{t_{i}} q_{i}\left(s-t_{i}\right) u(s, \xi) d s, \\
\Delta u^{\prime}\left(t_{i}\right)=\int_{-\infty}^{t_{i}} \bar{q}_{i}\left(s-t_{i}\right) u(s, \xi) d s, \quad i=1,2, \ldots, n,
\end{array}\right.
$$

where $h(\cdot) \in L^{\infty}(\mathbb{R}), \varphi \in C_{0} \times L^{2}(\rho, X), z \in X, 0<t_{1}<\cdots<t_{n}<b$ and

(i) The function $F: \mathbb{R}^{4} \rightarrow \mathbb{R}$ is continuous and there is a continuous function $\mu: \mathbb{R}^{2} \rightarrow \mathbb{R}$ such that $|F(t, s, \xi, x)| \leq \mu(t, s)|x|,(t, s, \xi, x) \in \mathbb{R}^{4}$.

(ii) The function $q_{i} \in C\left(\mathbb{R}^{+}, \mathbb{R}\right)$ and $c_{i}^{1}=\left(\int_{-\infty}^{0} q_{i}^{2}(\theta) \rho^{-1}(\theta) d \theta\right)^{\frac{1}{2}}<\infty, i=1,2, \ldots, n$.

(iii) The function $\bar{q}_{i} \in C\left(\mathbb{R}^{+}, \mathbb{R}\right)$ and $c_{i}^{2}=\left(\int_{-\infty}^{0} \bar{q}_{i}^{2}(\theta) \rho^{-1}(\theta) d \theta\right)^{\frac{1}{2}}<\infty, i=1,2, \ldots, n$.

(iv) The function $\varphi$ defined by $\varphi(\theta)(\xi)=: \phi(\theta, \xi)$ belongs to $\beta$.

Assuming that the conditions (i)-(iv) are satisfied, then system (32) can be modeled as the abstract impulsive Cauchy problem (1) by defining

$$
\begin{aligned}
& g_{1}(t, \varphi, x)(\xi)=\int_{-\infty}^{-t} h(s) \varphi(s, \xi) d s+x(\xi), \\
& g_{2}(t, \varphi)(\xi)=\int_{-\infty}^{0} F(t, s, \xi, \varphi(s, \xi)) d s,
\end{aligned}
$$




$$
\begin{aligned}
& I_{i}^{1}(\varphi)(\xi)=\int_{-\infty}^{0} q_{i}(s) \varphi(s, \xi) d s \quad(j=1,2), \\
& I_{i}^{2}(\varphi)(\xi)=\int_{-\infty}^{0} \bar{q}_{i}(s) \varphi(s, \xi) d s \quad(j=1,2),
\end{aligned}
$$

where

$$
x(\xi)=\int_{0}^{t} h(s-t) \varphi(0, \xi) d s, \quad k_{1}(t, s, \varphi)(\xi)=h(s-t) \varphi(0, \xi) .
$$

Moreover, $g_{1}(t, \cdot), I_{i}^{j}(\cdot)(i=1,2, \ldots, n, j=1,2)$ and $k_{1}(t, s, \cdot)$ are bounded linear operators, $\left\|I_{i}^{j}\right\| \leq c_{i}^{j}(i=1,2, \ldots, n, j=1,2)$,

$$
\begin{aligned}
& \left\|g_{1}(t, \varphi, x)\right\| \leq\left(\int_{-\infty}^{-t} a^{2}(s) \rho^{-1}(s) d s\right)^{\frac{1}{2}}\|\varphi\|_{\beta}+\|x\|, \\
& \left\|g_{2}(t, \varphi)\right\| \leq d(t)\|\varphi\|_{\beta}, \\
& \left\|k_{1}(t, s, \varphi)\right\| \leq\|a(\cdot)\|_{\infty}\|\varphi\|_{B}, \quad t \in[0, b],
\end{aligned}
$$

where $d(t)=\left(\int_{-\infty}^{0} \mu^{2}(t, s) \rho^{-1}(s) d s\right)^{\frac{1}{2}}$. If the cosine function family $C(t), t \in \mathbb{R}$, is equicontinuous, all the conditions of Theorem 3.2 are satisfied (see [1] for details), so system (32) has at least one mild solution. However, if we select $\rho(s)=e^{-s}, \bar{q}_{1}(s)=s$ in (iii), we have $c_{1}^{2}=\left(\int_{-\infty}^{0} s^{2} e^{s} d s\right)^{\frac{1}{2}}=\sqrt{2}$. But

$$
\left[1+\left(\int_{-b}^{0} \rho(t) d t\right)^{\frac{1}{2}}\right]\left[b \max \left\{\left(\int_{-\infty}^{-t} a^{2}(s) \rho^{-1}(s) d s\right)^{\frac{1}{2}}, 1\right\}+\sum_{i=1}^{n}\left(c_{i}^{1}+c_{i}^{2}\right)\right]>1,
$$

the restrictive conditions (3), (4) and (6) do not hold. Thus, our results are different from the corresponding known results.

Example 4.2 Consider the partial neutral functional integro-differential system:

$$
\left\{\begin{array}{l}
\frac{\partial}{\partial t}\left(\frac{\partial}{\partial t} u(t, \xi)+\int_{-\infty}^{t} \int_{0}^{\pi} b(s-t, \eta, \xi) u(s, \eta) d \eta d s\right) \\
\quad=\frac{\partial^{2}}{\partial \xi^{2}} u(t, \xi)+\int_{0}^{t} \int_{-\infty}^{0} \mu(s, \theta) u^{\prime}(s+\theta, \xi) d \theta d s \\
u(t, 0)=u(t, \pi)=0, \quad t \in J=[0, b] \\
u(\theta, \xi)=\varphi(\theta, \xi), \quad \theta \in(-\infty, 0], \xi \in[0, \pi] \\
\frac{\partial}{\partial t} u(\theta, \xi)=\psi(\theta, \xi), \quad \theta \in(-\infty, 0], \xi \in[0, \pi] \\
\Delta u\left(t_{i}\right)=\int_{-\infty}^{0} q_{i}(\theta) u^{\prime}\left(t_{i}+\theta, \xi\right) d \theta, \quad i=1,2, \ldots, n, \\
\Delta u^{\prime}\left(t_{i}\right)=\int_{-\infty}^{0} \bar{q}_{i}(\theta) u\left(t_{i}+\theta, \xi\right) d \theta, \quad i=1,2, \ldots, n,
\end{array}\right.
$$

where $\varphi, \psi \in C_{0} \times L^{2}(h, X), \varphi(0, \cdot) \in H^{1}([0, \pi]), 0<t_{1}<\cdots<t_{n}<b$ and

(v) The functions $b(\theta, \eta, \xi), \frac{\partial}{\partial \xi} b(\theta, \eta, \xi)$ are measurable, $b(\theta, \eta, 0)=b(\theta, \eta, \pi)=0$ and

$$
\begin{aligned}
& c=\max \left\{\left(\int_{0}^{\pi} \int_{-\infty}^{0} \int_{0}^{\pi} \frac{1}{\rho(\theta)}\left(\frac{\partial^{i} b(\theta, \eta, \xi)}{\partial \xi^{i}}\right)^{2} d \eta d \theta d \xi\right)^{\frac{1}{2}}: i=0,1\right\}<K_{b}^{-1}, \\
& \sup _{t \in J} \int_{0}^{\pi} \int_{0}^{t} \int_{0}^{\pi}\left(\frac{\partial}{\partial s} b(s-t, \eta, \xi)\right)^{2} d \eta d s d \xi<\infty .
\end{aligned}
$$


(vi) The function $\mu: \mathbb{R}^{2} \rightarrow \mathbb{R}$ is continuous and $\int_{-\infty}^{0} \mu^{2}(t, \theta) \rho^{-1}(\theta) d \theta=d(t)<\infty$.

Assuming that the conditions (ii)-(vi) are satisfied, system (33) can be modeled as the abstract Cauchy problem (2) by defining

$$
\begin{aligned}
& g(t, \varphi, \psi)(\xi)=\int_{-\infty}^{0} \int_{0}^{\pi} b(\theta, \eta, \xi) \varphi(\theta, \eta) d \eta d \theta, \\
& f(t, \varphi, \psi)(\xi)=\int_{-\infty}^{0} \mu(t, \theta) \psi(\theta, \xi) d \theta, \\
& I_{i}^{1}(\varphi, \psi)(\xi)=\int_{-\infty}^{0} q_{i}(\theta) \psi(\theta, \xi) d \theta \quad(j=1,2), \\
& I_{i}^{2}(\varphi, \psi)(\xi)=\int_{-\infty}^{0} \bar{q}_{i}(\theta) \varphi(\theta, \xi) d \theta \quad(j=1,2),
\end{aligned}
$$

where $\varphi, \psi \in \beta, I_{i}^{j}(\cdot)(i=1,2, \ldots, n, j=1,2), g(t, \cdot)$ are bounded linear operators and

$$
\|f(t, \varphi, \psi)\| \leq d(t)\left(\|\varphi\|_{\beta}+\|\psi\|_{\beta}\right), \quad d(t)=\left(\int_{-\infty}^{0} \mu^{2}(t, s) \rho^{-1}(s) d s\right)^{\frac{1}{2}}, \quad t \in J .
$$

Moreover, for every bounded set $Q \subset S^{1}(b)$, it follows from (34) and the proof in [26] that the set of functions $\left\{\left(\widetilde{\omega_{x}}\right)_{i}(t): x \in Q\right\}$ is uniformly equicontinuous on $\bar{J}_{i}=\left[t_{i}, t_{i+1}\right]$ for every $i=0,1, \ldots, n$. If the cosine function family $C(t), t \in \mathbb{R}$, is equicontinuous, all the conditions of Theorem 3.4 are satisfied, so system (33) has at least one mild solution.

Remark 4.3 From the results of this paper, we know that the compactness condition of the impulsive term, the restrictive conditions on a priori estimation and noncompactness measure estimation can be deleted for the existence results of abstract impulsive functional Volterra integro-differential equations Cauchy problems.

\section{Competing interests}

The author declares that they have no competing interests.

\section{Acknowledgements}

The author is grateful to the referees for their careful reading and helpful suggestions that have led to the present improved version of the original paper. The work was supported by the Natural Science Foundation of Anhui Province (11040606M01), Anhui Educational Committee (KJ2011A061, KJ2011B052), China.

Received: 5 April 2013 Accepted: 5 August 2013 Published: 9 September 2013

\section{References}

1. Hernández, EM, Rabelloa, M, Henríquez, HR: Existence of solutions for impulsive partial neutral functional differential equations. J. Math. Anal. Appl. 331, 1135-1158 (2007)

2. Chang, YK, Arjunam, MM, Kavitha, V: Existence results for a second order impulsive functional differential equation with sates-dependent delay. Diff. Eqs. \& Appl. 1(3), 325-339 (2009)

3. Balachandran, K, Annapoorani, N: Existence results for impulsive neutral evolution integro-differential equations with infinite delay. Nonlinear Analysis: Hybrid Systems. 3, 674-684 (2009)

4. Cuevas, C, Hernández, EM, Rabelo, M: The existence of solutions for impulsive neutral functional differential equations. Comput. Math. Appl. 58, 744-757 (2009)

5. Park, JY, Balachandran, K, Annapoorani, N: Existence results for impulsive neutral functional integrodifferential equations with infinite delay. Nonlinear Anal. 71, 3152-3162 (2009)

6. Rabelo, MN, Henrique, M, Siracusa, G: Existence of integro-differential solutions for a class of abstract partial impulsive differential equations. J. Inequalities and Appl. 135, 1-19 (2011)

7. Changa, YK, Angurajb, A, Arjunan, MM: Existence results for impulsive neutral functional differential equations with infinite delay. Nonlinear Analysis: Hybrid Systems. 2, 209-218 (2008)

8. Hernández, EM, Henríquez, HR, McKibben, MA: Existence results for abstract impulsive second-order neutral functional differential equations. Nonlinear Anal. 70, 2736-2751 (2009) 
9. Chalishajar, DN: Controllability of impulsive partial neutral functional differential equation with Infinite Delay. Int. J. Math. Anal. 5(8), 369-380 (2011)

10. Hernández, EM, Henríquez, HR, McKibben, MA: Existence of solutions for second order partial neutral functional differential equations. Integr. equ. oper. theory. 62, 191-217 (2008)

11. Hernández, EM, McKibben, MA Some comments on: Existence of solutions of abstract nonlinear second-order neutral functional integrodifferential equations. Comput. Math. Appl. 50, 655-669 (2005)

12. Hernandez, E, Balachandran, $\mathrm{K}$, Annapoorani, $\mathrm{N}$ : Existence results for a damped second order abstract functional differential equation with impulses. Mathematical and Computer Modelling. 50, 1583-1594 (2009)

13. Runping, Y: Impulsive partial neutral functional differential equation of second-order with infinite delay. Inter. J. Nonlinear Sci. 12(2), 245-256 (2011)

14. Vijayakumar, V, Sivasankarmn, S, Arjunan, MM: Existence of solutions for second-order impulsive neutral functional integro-differential equations with infinite delay. Nonlinear Stud. 19(2), 327-343 (2012)

15. Vijayakumar, V, Sivasankaran, S, Arjunan, MM: Existence of solutions for double perturbed impulsive neutral functional evolution equations. J. KSIAM. 15(4), 253-265 (2011)

16. Runping, Y: Existence of solutions for impulsive partial neutral functional differential equation with infinite delay. Nonlinear Anal. 73, 155-162 (2010)

17. Fattorini, HO: Second Order Linear Differential Equations in Banach Spaces. North-Holland Math. Stud., vol. 108 North-Holland, Amsterdam (1985)

18. Kisy'nski, J: On cosine operator functions and one parameter group of operators. Studia Math. 49, 93-105 (1972)

19. Hale, JK, Kato, J: Phase space for retarded equations with infinite delay. Funkcial Ekvac 21, 11-41 (1978)

20. Chalishajar, DN: Controllability of Second Order Impulsive Neutral Functional Differential Inclusions with Infinite Delay. J Optim Theory Appl. 154(2), 672-684 (2012)

21. Chalishajar, DN, Acharya, FS: Controllability of second order semi linear neutral impulsive differential inclusions on unbounded domain with infinite delay in Banach spaces. Bull. Korean Math. Soc. 48(4), 813-838 (2011)

22. Dajun, G, Lakshmikantham, V, Liu, X: Nonlinear Integral Equations in Abstract Spaces. Kluwer Academic, Dordrecht (1996)

23. Henríquez, HR, Vásquez, CH: Differentiability of solutions of second-order functional differential equations with unbounded delay. J. Math. Anal. Appl. 280(2), 284-312 (2003)

24. Heine, HP: On the behavior of measure of noncompactness with respect to differentiation and integration of vector valued functions. Nonlinear Anal. 7, 1351-1371 (1983)

25. Mönch, H: Boundary value problems for nonlinear ordinary equations of second order in Banach spaces. Nonlinear Anal. 4, 985-999 (1980)

26. Hernández, E: Existence results for partial neutral functional integro-differential equations with unbounded delay. J. Math. Anal. Appl. 292, 194-210 (2004)

doi:10.1186/1687-2770-2013-203

Cite this article as: Xie: Solvability of impulsive partial neutral second-order functional integro-differential equations with infinite delay. Boundary Value Problems 2013 2013:203.

\section{Submit your manuscript to a SpringerOpen ${ }^{\circ}$ journal and benefit from:}

- Convenient online submission

Rigorous peer review

- Immediate publication on acceptance

- Open access: articles freely available online

- High visibility within the field

- Retaining the copyright to your article 